\title{
Ascorbic acid as antihypertensive agent
}

Omar Yacoub ${ }^{1}$, Mohammad Altamimi ${ }^{*}$.

${ }^{1}$ Department of Nutrition and Food Technology, Faculty of Agriculture and Veterinary Medicine, An-Najah National University, Nablus, Palestine

*Corresponding Author: Mohammad Altamimi, Email: m.altamimi@najah.edu,

Tel: +97(0)595591255.

Citation: Mohammad Altamimi, Omar Yacoub (2016) Ascorbic acid as antihypertensive agent. Int J Nutritional Science \& Food Technology 2:2, 44-47

Copyright: (C) 2016 Mohammad Altamimi et al. This is an open-access article distributed under the terms of the Creative Commons Attribution License, which permits unrestricted use, distribution, and reproduction in any medium, provided the original author and source are credited.

Received August 2, 2016; Accepted September 20, 2016; Published September 30, 2016.

\section{Abstract}

Vitamin C (Ascorbic acid) is a water-soluble vitamin and is highly polar compound. Humans have to get what they need of Vitamin $\mathrm{C}$ from food, including citrus fruits, broccoli, and tomatoes. Human bodies require vitamin $\mathrm{C}$ for the growth and repair of tissues in all parts of the body. Vitamin $\mathrm{C}$ is one of many antioxidants that block some of the damage caused by free radicals. There are some thoughts that vitamin $\mathrm{C}$ might help the heart and the blood vessels by preventing clots in veins and arteries, high blood pressure, and high cholesterol. Essential hypertension is characterized by endothelial dysfunction, arterial stiffness, and increased oxidative stress. This mini review will focus on the role of vitamin $\mathrm{C}$ in these major symptoms.

Key Words Ascorbic acid, LDL oxidation, cell adhesion, Endothelium, Oxidative stress.

\section{Introduction}

Vitamin C is sensitive to light, air, and heat, so you'll get the most vitamin $C$ if you eat fruits and vegetables raw or lightly cooked. The best way to get the daily requirement of essential vitamins, including vitamin $\mathrm{C}$, is to eat a balanced diet that contains a variety of foods (1).

The presumption that oxidative stress, amongst several other factors, plays an important role in atherogenesis implies that the development and progression of atherosclerosis can be inhibited by antioxidants (2). In this review we discussed several mechanisms by which the antioxidant ascorbate (vitamin C) affects atherosclerosis. These mechanisms include inhibition of LDL oxidation, inhibition of leukocyte adhesion to the endothelium and vascular endothelial dysfunction.

\section{Ascorbic acid metabolism}

Ascorbate donates a single electron to become the ascorbate radical, which reacts with another ascorbate radical to form a molecule each of ascorbate and dehydroascorbate (DHA). The latter is unstable at physiologic $\mathrm{pH}$ and if not reduced back to ascorbate via GSH-dependent mechanisms. It will undergo irreversible ring opening and loss. In buffers, DHA forms a hemiketal that has a molecular structure resembling that of glucose (3).

\section{Oxidative stress}

Oxidative stress (free radicals generating process) is thought to play an important role in atherosclerotic vascular disease (4). Thus, dietary antioxidants such as ascorbate (vitamin C) and polyphenols can protect against the development and progression of atherosclerosis in experimental models (4). Numerous observational studies have shown an inverse association between antioxidant intake or body status and the risk of cardiovascular diseases (5).

\section{LDL Oxidation}

Oxidatively modified LDL has been implicated in the pathogenesis of atherosclerosis (4 and 5) Although the mode of LDL oxidation in vivo is incompletely understood, the mechanisms of LDL oxidation in vitro have been studied extensively (4). Modification of the protein moiety of LDL (apolipoprotein B-100), either directly by leukocyte-derived oxidants such as hypochlorous acid 
or indirectly by lipid hydroperoxide breakdown products such as 4-hydroxynonenal and malondialdehyde, results in a form of LDL that is internalized by macrophages via the scavenger receptor pathway leading to foam cell formation. Although redox-active transition metal ions seem to play a pivotal role in cell-mediated LDL oxidation (4). Various metal ion-independent mechanisms of LDL oxidation have been proposed, such as reactive nitrogen and chlorine species. Furthermore, there is convincing evidence that in vitro lipid peroxidation in LDL is initiated by $\alpha$-tocopheroxyl radicals formed in the lipoprotein on attack by free radicals or other reactive species. Thus, $\alpha$-tocopherol can act as a pro-oxidant, rather than an antioxidant, in LDL incubated in vitro (6 and 7)

\section{Ascorbate and LDL Oxidation}

Human plasma and other extracellular fluids contain numerous water-soluble antioxidants, including ascorbate, urate, bilirubin, and various thiol compounds (8 and 9). Experimental data on the effects of vitamin $\mathrm{C}$ supplementation of human subjects on ex vivo LDL oxidation are sparse, mainly because ascorbate is removed from LDL during isolation from plasma. However, there is convincing evidence from in vitro studies that physiological concentrations of ascorbate strongly inhibit LDL oxidation by vascular cells and neutrophils, as well as in cell-free systems (10).

Ascorbate prevents oxidative modification of LDL primarily by scavenging free radicals and other reactive species in the aqueous milieu. Thus, direct and rapid trapping of these aqueous reactive species by ascorbate prevents them from interacting with and oxidizing LDL. Ascorbyl radicals formed in this process may be reduced back to ascorbate by dismutation, chemical reduction (eg, by glutathione), or enzymatic reduction (eg, by thioredoxin reductase) (11). Dismutation also produces dehydroascorbic acid (ADA), which in turn can be reduced back to ascorbate by glutathione, thioredoxin reductase, and glutaredoxin. Ascorbate can also prevent the pro-oxidant activity of $\alpha$-tocopherol by reducing the $\alpha$-tocopheroxyl radical to $\alpha$-tocopherol, thereby acting as a "coantioxidant" and inhibiting LDL oxidation.

\section{Cell Adhesion}

Adhesion of leukocytes to the endothelium is an important initiating step in atherogenesis (12-14). Various studies have shown that monocytes bind selectively to aortic pre lesion areas and atherosclerotic lesions, which also exhibit increased expression of adhesion molecules compared with normal tissue(13). Cultured endothelial cells exposed to inflammatory cytokines or oxidized LDL exhibit enhanced expression of cell adhesion molecules, such as intercellular adhesion molecule-1 (ICAM-1), vascular cell adhesion molecule-1 (VCAM-1), and E-selectin (14). These adhesion molecules interact with specific ligands expressed on the surface of leukocytes, such as the $\beta 1$ and $\beta 2$ integrins, and mediate leukocyte rolling, firm attachment to the endothelium, and subsequent migration into the subendothelial space (14).

\section{Ascorbate and Cell Adhesion}

Two recent human studies have investigated the role of ascorbate in inhibiting cell-cell adhesion (15 and 16). Smokers have decreased plasma levels of ascorbate, and monocytes isolated from smokers exhibit increased adhesion to cultured endothelial cells compared with monocytes isolated from nonsmokers. Supplementation of smokers with $2 \mathrm{~g}$ /day of vitamin $\mathrm{C}$ for 10 days elevated plasma ascorbate levels almost 2 -fold and significantly reduced monocyte adhesion to cultured endothelial cells (15). This finding indicates that upregulation of ligands on monocytes is inhibited by ascorbate. In another study, however, supplementation of smokers with $2 \mathrm{~g}$ of vitamin C 2 hours before isolation of monocytes had no effect on ex vivo monocyte-endothelial cell adhesion or endothelial ICAM1 surface expression, despite a $>3$-fold increase in serum ascorbate levels. The supplementation period in this study may have been too short to affect intracellular ascorbate levels.

Several in vivo studies using intravital microscopy in hamsters have demonstrated an important role of ascorbate in inhibiting leukocyte-endothelial cell interactions induced by cigarette smoke or oxidized LDL, likely by antioxidant mechanisms. Lehr et al., (1997) showed that the induction of leukocyte adhesion to the vascular wall elicited by cigarette smoke is due to the formation of oxidatively modified lipids with platelet-activating factor-like activity. Administration of ascorbate prevented the accumulation of these platelet-activating factor-like lipids and the subsequent leukocyte-endothelial cell interactions (17).

\section{Endothelium Nitric Oxide (NO) synthesis}

Endothelium-derived NO (EDNO) is a pivotal molecule in the regulation of vascular tone and homeostasis (18). In addition to stimulating vascular smooth muscle cell relaxation and vasodilation, EDNO exerts a number of potent antiatherogenic effects, including inhibition of smooth muscle cell proliferation, platelet aggregation, and leukocyte-endothelial cell interactions (18). EDNO is synthesized from L-arginine through the action of constitutive and inducible isoforms of the NADPH-dependent enzyme NO synthase. The enzyme requires a number of cofactors, including flavin adenine dinucleotide, flavin mononucleotide, tetrahydrobiopterin, and possibly thiols (19). Endothelial vasodilator dysfunction has been observed in patients with coronary artery disease or subjects with coronary risk factors. Most of these conditions are associated with increased oxidative stress, particularly increased production of superoxide radicals, which can inactivate EDNO (20). In addition, oxidized LDL has been shown to inhibit the synthesis of EDNO or attenuate its biological activity (21).

\section{Ascorbate and Endothelium NO synthesis}

Numerous clinical studies have consistently demonstrated beneficial effects of vitamin $\mathrm{C}$ treatment on endotheliumdependent vasodilation in individuals with coronary artery disease or coronary risk factors (22).

There are a number of potential mechanisms underlying the salubrious effects of ascorbate on endothelial function. First, ascorbate may be decreasing the levels of superoxide radicals and oxidized LDL, both of which react with and inactivate NO. Because of the facile reaction between superoxide and NO radicals, 
relatively high concentrations of ascorbate $(\approx 10 \mathrm{mmol} / \mathrm{L})$ are required to effectively inhibit the reaction of $\mathrm{NO}$ with superoxide. Such concentrations are potentially achievable in plasma by intraarterial infusion or in the cytoplasm as a result of cellular uptake of ascorbate. Second, Ascorbate may indirectly enhance endotheliumdependent vasodilation by sparing intracellular thiols, which in turn stabilize EDNO through the formation of biologically active S-nitrosothiols. Reducing agents such as ascorbate have also been implicated in the rapid release of NO from S-nitrosothiols. Finally, Heller et al., 1999 (23) and, more recently, Huang et al., 2000 (24) have shown that physiological concentrations of ascorbate increase the synthesis and biological activity of NO in cultured endothelial cells by increasing intracellular tetrahydrobiopterin. Thus, a very likely mechanism by which intracellular ascorbate stimulates NOS activity is regeneration of tetrahydrobiopterin from the trihydrobiopterin radical. Such a mechanism of action of ascorbate would also prevent NOS from leaking superoxide radicals (25).

\section{Conclusion}

From epidemiological studies people who eat foods rich in vitamin $\mathrm{C}$ have a lower risk of high blood pressure than people who have poorer diets. Eating foods rich in vitamin $\mathrm{C}$ is important for overall health, especially when person is at risk for high blood pressure. Nutritionists and dieticians' most frequently recommended treatment and prevention of high blood pressure is known as the DASH (Dietary Approaches to Stop Hypertension) diet which includes lots of fruits and vegetables loaded with vitamin $\mathrm{C}$.

\section{References}

1. Slavin J. L. and Lloyd B. (2012). Health Benefits of Fruits and Vegetables . Adv. Nutr. 3: 506-16.

2. Covas M.I., Nyyssönen K., Poulsen H.E., Kaikkonen J., Zunft H.J., Kiesewetter H., Gaddi A., de la Torre R., Mursu J., Bäumler H., Nascetti S., Salonen J.T., Fitó M., Virtanen J., and Marrugat J. (2006). The effect of polyphenols in olive oil on heart disease risk factors: a randomized trial. Ann Intern Med. 145 (5):333-41.

3. May J. M. and Harrison F. E. (2013). Role of Vitamin C in the Function of the Vascular Endothelium. Antioxidants \& Redox Signaling. 19 (17) : 2068-83.

4. Berliner J.A. and Heinecke J.W. (1996). The role of oxidized lipoproteins in atherogenesis. Free Radic Biol Med. 20:707-27.

5. Diaz M.N., Frei B., Vita J.A. and Keaney J.F. (1997). Antioxidants and atherosclerotic heart disease. N Engl J Med. 337:408 -16.

6. Bowry V.W. and Stocker R. (1993). Tocopherol-mediated peroxidation: the prooxidant effect of vitamin $\mathrm{E}$ on the radicalinitiated oxidation of human low-density lipoprotein. J Am Chem Soc. 115:6029-44.

7. Neuzil J., Thomas S.R. and Stocker R. (1997). Requirement for, promotion, or inhibition By a-tocopherol of radical-induced initiation of plasma lipoprotein lipid peroxidation. Free Radic Biol Med. 22:57-71.
8. Frei B., England L., Ames B.N. (1989). Ascorbate is an outstanding antioxidant in human blood plasma. Proc Natl Acad Sci U S A .86:6377- 81 .

9. Carr A.C., Tijerina T. and Frei B. (2000). Vitamin C protects against and reverses specific hypochlorous acid- and chloraminedependent modifications of low-density lipoprotein. Biochem J. 346:491- 99 .

10. Retsky K.L., Freeman M.W. and Frei B. (1993). Ascorbic acid oxidation product(s) protect human low density lipoprotein against atherogenic modification: anti- rather than prooxidant activity of vitamin $\mathrm{C}$ in the presence of transition metal ions. J Biol Chem. 268:1304-09.

11. May J.M., Cobb C.E., Mendiratta S., Hill K.E. and Burk R.F.(1998) Reduction of the ascorbyl free radical to ascorbate by thioredoxin reductase. J Biol Chem .273:23039 -45.

12. Poston R.N. and Johnson-Tidey R.R. (1996). Localized adhesion of monocytes to human atherosclerotic plaques demonstrated in vitro: implications for atherogenesis. Am J Pathol. $149: 73-80$.

13. Davies M.J., Gordon J.L., Gearing A.J., Pigott R., Woolf N., Katz D., and Kyriakopoulos A. (1993).The expression of the adhesion molecules ICAM-1, VCAM-1, PECAM, and E-selectin in human atherosclerosis. J Pathol. 171:223-29.

14. Panes J., Perry M. and Granger D.N. (1999). Leukocyteendothelial cell adhesion: avenues for therapeutic intervention. $\mathrm{Br}$ J Pharmacol. 126:537-50.

15. Weber C., Wolfgang E., Weber K. and Weber P.C. (1996). Increased adhesiveness of isolated monocytes to endothelium is prevented by vitamin $\mathrm{C}$ intake in smokers. Circulation. 93:1488 -92 .

16. Adams M.R., Jessup W. and Celermajer D.S. (1997). Cigarette smoking is associated with increased human monocyte adhesion to endothelial cells: reversibility with oral L-arginine but not vitamin C. J Am Coll Cardiol . 29:491- 97.

17. Lehr H.A., Weyrich A.S., Saetzler R.K., Jurek A., Arfors K.E., Zimmerman G.A., Prescott S.M. and McIntyre T.M. (1997). Vitamin C blocks inflammatory platelet-activating factor mimetics created by cigarette smoking. J Clin Invest. 99:2358 -64.

18. Furchgott R.F. (1996). The discovery of endothelium-derived relaxing factor and its importance in the identification of nitric oxide. JAMA. 276: 1186-1188.

19. Moncada S. and Higgs A. (1993). The L-arginine-nitric oxide path way. N Engl J Med .329:2002-12.

20. Gryglewski R.J., Palmer R.M. and Moncada S. (1986). Superoxide anion is involved in the breakdown of endotheliumderived vascular relaxing factor. Nature. 320:454 -56.

21. Chin J.H., Azhar S. and Hoffman B.B. (1992). Inactivation of endothelial derived relaxing factor by oxidized lipoproteins. J Clin Invest .89:10-18. 
22.Carr A.C., McCall M.R., Frei B. (2000). Oxidation of LDL by myeloperoxidase and reactive nitrogen species: reaction pathways and antioxidant protection. Arterioscler Thromb Vasc Biol. 20:1716-1723.

23. Heller R., Munscher-Paulig F., Grabner R. and Till U. (1999). L-Ascorbic acid potentiates nitric oxide synthesis in endothelial cells. J Biol Chem. 274: 8254-60.
24. Huang A., Vita J.A., Venema R.C. and Keaney J.F. (2000). Ascorbic acid enhances endothelial nitric oxide synthase activity by increasing intracellular tetrahydrobiopterin. J Biol Chem .275:17399-17406.

25. Green D., O’Driscoll G., Rankin J.M., Maiorana A.J. and Taylor R.R. (1998). Beneficial effect of vitamin E administration on nitric oxide function in subjects with hypercholesterolaemia. Clin Sci (Colch). 95:361-67. 